\title{
On the solutions of two special types of Riccati difference equation via Fibonacci numbers
}

Durhasan T Tollu', Yasin Yazlik ${ }^{2^{*}}$ and Necati Taskara ${ }^{3}$

\section{"Correspondence:}

yyazlik@nevsehir.edu.tr

${ }^{2}$ Nevsehir University, Department of

Mathematics, Faculty of Science

and Art, Nevsehir, Turkey

Full list of author information is

available at the end of the article

\begin{abstract}
In this study, we investigate the solutions of two special types of the Riccati difference equation $x_{n+1}=\frac{1}{1+x_{n}}$ and $y_{n+1}=\frac{1}{-1+y_{n}}$ such that their solutions are associated with Fibonacci numbers.

MSC: 11B39; 39A 10; 39A 13

Keywords: Riccati difference equation; equilibrium point; Fibonacci numbers; golden ratio
\end{abstract}

\section{Introduction}

Nonlinear difference equations have long interested researchers in the field of mathematics as well as in other sciences. They play a key role in many applications such as the natural model of a discrete process. There have been many recent investigations and interest in the field of nonlinear difference equations by several authors [1-14]. For example, in [1], Brand defined a sequence which stems from the Riccati difference equation

$$
x_{n+1}=\frac{a x_{n}+b}{c x_{n}+d}
$$

In [6], Cinar studied the solution of the difference equation

$$
x_{n+1}=\frac{x_{n-1}}{1+x_{n} x_{n-1}} .
$$

In [7], Papaschinopoulos and Papadopoulos studied the fuzzy difference equation

$$
x_{n+1}=A+\frac{B}{x_{n}},
$$

which is a special case of the Riccati difference equation. In [8], Elabbasy et al. obtained the Fibonacci sequence in solutions of some special cases of the following difference equation

$$
x_{n+1}=\frac{a x_{n-l} x_{n-k}}{b x_{n-p}-c x_{n-q}} .
$$

(0) 2013 Tollu et al.; licensee Springer. This is an Open Access article distributed under the terms of the Creative Commons Attribution License (http://creativecommons.org/licenses/by/2.0), which permits unrestricted use, distribution, and reproduction in any medium, provided the original work is properly cited. 
In [9], the author deals with behavior of the solution of the nonlinear difference equation

$$
x_{n+1}=a x_{n-1}+\frac{b x_{n} x_{n-1}}{c x_{n}+d x_{n-2}} .
$$

Also, he gives specific forms of the solutions of four special cases of this equation. These specific forms also contain Fibonacci numbers.

Fibonacci numbers have been interesting to the researchers for a long time to get the main theory and applications of these numbers. For instance, the ratio of two consecutive Fibonacci numbers converges to the golden section $\alpha=\frac{1+\sqrt{5}}{2}$. The applications of the golden ratio appear in many research areas, particularly in physics, engineering, architecture, nature and art. Physicists Naschie and Marek-Crnjac gave some examples of the golden ratio in theoretical physics and physics of high energy particles [15-19]. We should recall that the Fibonacci sequence $\left\{F_{n}\right\}_{n=0}^{\infty}$ has been defined by the recursive equation

$$
F_{n+2}=F_{n+1}+F_{n}
$$

with initial conditions $F_{0}=0, F_{1}=1$. Also, it is obtained to extend the Fibonacci sequence backward as

$$
F_{-n}=F_{-n+2}-F_{-n+1}=(-1)^{n+1} F_{n} .
$$

One can clearly obtain the characteristic equation of (1) as the form $x^{2}-x-1=0$ such that the roots

$$
\alpha=\frac{1+\sqrt{5}}{2} \text { and } \beta=\frac{1-\sqrt{5}}{2} .
$$

Hence the Binet formula for Fibonacci numbers

$$
F_{n}=\frac{\alpha^{n}-\beta^{n}}{\alpha-\beta},
$$

can be thought of as a solution of the recursive equation in (1). Also, the following ratio is satisfied:

$$
\lim _{n \rightarrow \infty} \frac{F_{n+r}}{F_{n}}=\alpha^{r}
$$

where $r \in \mathbb{Z}$.

Let us consider the following lemma which will be needed for the results in this study.

Lemma 1 [15] The following equalities hold:

(i) For $n>k+1, n \in \mathbb{N}^{+}$and $k \in \mathbb{N}, F_{n}=F_{k+1} F_{n-k}+F_{k} F_{n-(k+1)}$.

(ii) For $n>0, \alpha^{n}=\alpha F_{n}+F_{n-1}$ and $\beta^{n}=\beta F_{n}+F_{n-1}$.

(iii) For $n>0, F_{n-1} F_{n+1}-F_{n}^{2}=(-1)^{n}$ (Cassini's formula).

In this study, we consider the Riccati difference equation

$$
x_{n+1}=\frac{a+b x_{n}}{c+d x_{n}}, \quad n=0,1, \ldots
$$


Obviously, by taking $a=c=d=1, b=0$ and $a=d=1, c=-1, b=0$, equation (6), respectively, is transformed into the following equations:

$$
\begin{aligned}
& x_{n+1}=\frac{1}{1+x_{n}}, \quad n=0,1, \ldots, \\
& y_{n+1}=\frac{1}{-1+y_{n}}, \quad n=0,1, \ldots,
\end{aligned}
$$

where initial conditions are $x_{0} \in \mathbb{R}-\left\{-\frac{F_{m+1}}{F_{m}}\right\}_{m=1}^{\infty}$ and $y_{0} \in \mathbb{R}-\left\{\frac{F_{m+1}}{F_{m}}\right\}_{m=1}^{\infty}$, respectively, and $F_{m}$ is the $m$ th Fibonacci number.

The aim of this study is to investigate some relationships both between Fibonacci numbers and solutions of equations (7) and (8) and between the golden ratio and equilibrium points of equations (7) and (8).

\section{Main results}

Firstly, it is not difficult to prove that equilibrium points of equations (7) and (8) are $\bar{x}_{1}=$ $-\beta, \bar{x}_{2}=-\alpha$ and $\bar{y}_{1}=\alpha, \bar{y}_{2}=\beta$, respectively, where $\alpha=\frac{\sqrt{5}+1}{2}$ is the golden ratio and $\beta=\frac{1-\sqrt{5}}{2}$ is the conjugate of $\alpha$. Note that one of the equilibrium points of equation (8) is the golden ratio.

Theorem 1 For $n=0,1,2, \ldots$, the solutions of equations (7) and (8) are as follows:

(i) For $x_{0} \in \mathbb{R}-\left(\left\{\frac{1}{\alpha}, \frac{1}{\beta}\right\} \cup\left\{-\frac{F_{m+1}}{F_{m}}\right\}_{m=1}^{\infty}\right), x_{n}=\frac{F_{n}+F_{n-1} x_{0}}{F_{n+1}+F_{n} x_{0}}$.

(ii) For $y_{0} \in \mathbb{R}-\left(\{\alpha, \beta\} \cup\left\{\frac{F_{m+1}}{F_{m}}\right\}_{m=1}^{\infty}\right), y_{n}=\frac{F_{-n}+F_{-(n-1)} y_{0}}{F_{-(n+1)}+F_{-n} y_{0}}$.

Proof Firstly, in here we will just prove (ii) since (i) can be thought in the same manner.

(ii) We will prove this theorem by induction. For $k=0$,

$$
\frac{F_{0}+F_{1} y_{0}}{F_{-1}+F_{0} y_{0}}=\frac{0+1 y_{0}}{1+0 y_{0}}=y_{0} .
$$

Now assume that

$$
y_{k}=\frac{F_{-k}+F_{-(k-1)} y_{0}}{F_{-(k+1)}+F_{-k} y_{0}}
$$

is true for all positive integers $k$. Therefore, we have to show that it is true for $k+1$. Taking into account (2) and (9), we write

$$
\begin{aligned}
y_{k+1} & =\frac{1}{-1+y_{k}} \\
& =\frac{F_{-(k+1)}+F_{-k} y_{0}}{F_{-k}-F_{-(k+1)}+\left(F_{-(k-1)}-F_{-k}\right) y_{0}} \\
& =\frac{F_{-(k+1)}+F_{-k} y_{0}}{F_{-(k+2)}+F_{-(k+1)} y_{0}},
\end{aligned}
$$

which ends the induction and the proof.

Theorem 2 Let the solutions of equations (7) and (8) be $\left\{x_{n}\right\}_{n=0}^{\infty}$ and $\left\{y_{n}\right\}_{n=0}^{\infty}$, respectively and $x_{0} \in \mathbb{R}-\left\{-\frac{F_{m+1}}{F_{m}}\right\}_{m=1}^{\infty}$. Therefore, $\left\{x_{n}\right\}_{n=0}^{\infty}=\left\{-y_{n}\right\}_{n=0}^{\infty}$ is satisfied if and only if the initial conditions are $x_{0}=-y_{0}$. 
Proof First, assume that $\left\{x_{n}\right\}_{n=0}^{\infty}=\left\{-y_{n}\right\}_{n=0}^{\infty}$. Taking into account (2), we can write

$$
\begin{aligned}
\frac{F_{n}+F_{n-1} x_{0}}{F_{n+1}+F_{n} x_{0}} & =-\frac{F_{-n}+F_{-(n-1)} y_{0}}{F_{-(n+1)}+F_{-n} y_{0}} \\
& =\frac{F_{n}-F_{n-1} y_{0}}{F_{n+1}-F_{n} y_{0}} .
\end{aligned}
$$

By using simple mathematical operations and the well-known Cassini's formula for Fibonacci numbers, we have

$$
\begin{aligned}
& \left(F_{n-1} F_{n+1}-F_{n}^{2}\right) x_{0}=\left(F_{n}^{2}-F_{n-1} F_{n+1}\right) y_{0}, \\
& (-1)^{n} x_{0}=(-1)^{n+1} y_{0} \\
& x_{0}=-y_{0} .
\end{aligned}
$$

Second, assume that $x_{0}=-y_{0}$. By considering the solutions of equation (7), we get

$$
\begin{aligned}
x_{n} & =\frac{F_{n}+F_{n-1} x_{0}}{F_{n+1}+F_{n} x_{0}} \\
& =\frac{(-1)^{n+1} F_{n}-(-1)^{n+1} F_{n-1} y_{0}}{(-1)^{n+1} F_{n+1}-(-1)^{n+1} F_{n} y_{0}} \\
& =\frac{F_{-n}+F_{-(n-1)} y_{0}}{-F_{-(n+1)}-F_{-n} y_{0}} \\
& =\frac{F_{-n}+F_{-(n-1)} y_{0}}{-\left(F_{-(n+1)}+F_{-n} y_{0}\right)} \\
& =-y_{n},
\end{aligned}
$$

which is desired.

\section{Theorem 3 The following statements hold:}

(i) For the initial condition $x_{0}=\frac{1}{\alpha}\left(\right.$ or $\left.x_{0}=\frac{1}{\beta}\right)$, equation (7) has the fixed solution $x_{n}=\frac{1}{\alpha}$ $\left(\right.$ or $\left.x_{n}=\frac{1}{\beta}\right)$.

(ii) For the initial condition $y_{0}=\alpha$ (or $y_{0}=\beta$ ), equation (8) has the fixed solution $y_{n}=\alpha$ $\left(\right.$ or $\left.y_{n}=\beta\right)$.

Proof Here we will just prove (i) since the proof of (ii) can be done quite similarly.

(i) Firstly, let $x_{0}=\frac{1}{\alpha}=\frac{\sqrt{5}-1}{2}$ be the initial condition of equation (7). Then, by using Lemma 1(ii), we have

$$
x_{n}=\frac{F_{n}+\frac{F_{n-1}}{\alpha}}{F_{n+1}+\frac{F_{n}}{\alpha}}=\frac{\alpha F_{n}+F_{n-1}}{\alpha F_{n+1}+F_{n}}=\frac{\alpha^{n}}{\alpha^{n+1}}=\frac{1}{\alpha} .
$$

Secondly, let $x_{0}=\frac{1}{\beta}=-\frac{\sqrt{5}+1}{2}$ be the initial condition of equation (7). Then, by considering Lemma 1(ii), we obtain

$$
x_{n}=\frac{F_{n}+F_{n-1} x_{0}}{F_{n+1}+F_{n} x_{0}}=\frac{F_{n}+\frac{F_{n-1}}{\beta}}{F_{n+1}+\frac{F_{n}}{\beta}}=\frac{\beta F_{n}+F_{n-1}}{\beta F_{n+1}+F_{n}}=\frac{\beta^{n}}{\beta^{n+1}}=\frac{1}{\beta},
$$

which is desired. 
Theorem 4 The following statements hold:

(i) For $x_{0} \in \mathbb{R}-\left(\left\{\frac{1}{\beta}\right\} \cup\left\{-\frac{F_{m+1}}{F_{m}}\right\}_{m=1}^{\infty}\right)$, all the solutions of equation (7) converge to $-\beta$, where $\beta=\frac{1-\sqrt{5}}{2}$. That is, $\lim _{n \rightarrow \infty} x_{n}=-\beta$.

(ii) For $y_{0} \in \mathbb{R}-\left(\{\alpha\} \cup\left\{\frac{F_{m+1}}{F_{m}}\right\}_{m=1}^{\infty}\right)$, all the solutions of equation (8) converge to $\beta$, where $\beta=\frac{1-\sqrt{5}}{2}$. That is, $\lim _{n \rightarrow \infty} y_{n}=\beta$.

Proof To prove, we use the solutions of (7) and (8).

(i) By using Theorem 1(i), we can write

$$
\lim _{n \rightarrow \infty} x_{n}=\lim _{n \rightarrow \infty} \frac{F_{n}+F_{n-1} x_{0}}{F_{n+1}+F_{n} x_{0}}=\lim _{n \rightarrow \infty} \frac{1+\frac{F_{n-1}}{F_{n}} x_{0}}{\frac{F_{n+1}}{F_{n}}+x_{0}} .
$$

Thus, from (5), we have

$$
\lim _{n \rightarrow \infty} x_{n}=\frac{1+\frac{1}{\alpha} x_{0}}{\alpha+x_{0}}=\frac{1}{\alpha}=-\beta .
$$

(ii) The proof can be seen easily in a similar manner to Theorem 4(i).

Theorem 5 Let $\left\{x_{n}\right\}_{n=0}^{\infty}$ be the solution of (7). Then, we have

$$
\lim _{n \rightarrow \infty} \prod_{i=0}^{n} x_{i}=F_{0}
$$

Proof For $x_{0}=F_{0}$, the result is trivial. If $x_{0} \neq F_{0}$, by Theorem 1 , then we can write

$$
\begin{aligned}
& x_{0}=\frac{F_{0}+F_{-1} x_{0}}{F_{1}+F_{0} x_{0}}, \\
& x_{1}=\frac{F_{1}+F_{0} x_{0}}{F_{2}+F_{1} x_{0}}, \\
& \vdots \\
& x_{n}=\frac{F_{n}+F_{n-1} x_{0}}{F_{n+1}+F_{n} x_{0}} .
\end{aligned}
$$

By multiplying both sides of the above equalities, we obtain

$$
\prod_{i=0}^{n} x_{i}=\frac{F_{0}+F_{-1} x_{0}}{F_{n+1}+F_{n} x_{0}}=\frac{x_{0}}{F_{n+1}+F_{n} x_{0}} .
$$

Letting $n \rightarrow \infty$, the last equality gives the following result

$$
\lim _{n \rightarrow \infty} \prod_{i=0}^{n} x_{i}=F_{0}
$$

Consequently, the proof is completed.

The following theorem establishes that the Fibonacci numbers can be obtained by using the solutions of (7). 
Theorem 6 Let the initial condition of equation (7) be $x_{0}=\frac{F_{k}}{F_{k+1}}$, where $F_{k}$ is the kth Fibonacci number. For $n>k+1$ and $k, n \in \mathbb{Z}^{+}$, we have

$$
F_{n}=\frac{F_{k+1}}{x_{1} x_{2} \cdots x_{n-(k+1)}} .
$$

Proof Firstly, taking $n-(k+1)$ instead of $n$ in (10), we obtain

$$
\prod_{i=0}^{n-(k+1)} x_{i}=\frac{x_{0}}{F_{n-k}+F_{n-(k+1)} x_{0}} .
$$

Secondly, dividing both sides in (11) with $x_{0}$, we get

$$
\prod_{i=1}^{n-(k+1)} x_{i}=\frac{1}{F_{n-k}+F_{n-(k+1)} x_{0}} .
$$

Finally, by considering Lemma 1(i), we obtain

$$
\begin{aligned}
x_{1} x_{2} \cdots x_{n-(k+1)} & =\frac{1}{F_{n-k}+F_{n-(k+1)} x_{0}} \\
& =\frac{1}{F_{n-k}+F_{n-(k+1)} \frac{F_{k}}{F_{k+1}}} \\
& =\frac{F_{k+1}}{F_{n}},
\end{aligned}
$$

from which the result follows.

\section{Conclusion}

In this study, we mainly obtained the relationship between the solutions of Riccati difference equations (given in (7), (8)) and Fibonacci numbers. We also presented that the nontrivial solutions of equations in (7) and (8) actually converge to $-\beta$ and $\beta$, respectively, so that $\beta$ is conjugate to the golden ratio. We finally note that the results in this paper are given in terms of Fibonacci numbers.

\section{Competing interests}

The authors declare that they have no competing interests.

\section{Authors' contributions}

All authors completed the paper together. All authors read and approved the final manuscript.

\section{Author details}

${ }^{1}$ Necmettin Erbakan University, Department of Mathematics and Computers, Science Faculty, Konya, Turkey. ${ }^{2}$ Nevsehir University, Department of Mathematics, Faculty of Science and Art, Nevsehir, Turkey. ${ }^{3}$ Selcuk University, Department of Mathematics, Science Faculty, Konya, Turkey.

Received: 26 April 2013 Accepted: 28 May 2013 Published: 18 June 2013

\section{References}

1. Brand, L: A sequence defined by a difference equation. Am. Math. Mon. 62, 489-492 (1955)

2. Agarwal, RP: Difference Equations and Inequalities, 1st edn. Dekker, New York (1992) (2nd ed., (2000))

3. Gibbons, CH, Kulenović, MRS, Ladas, G: On the recursive sequence $x_{n+1}=\frac{\alpha+\beta x_{n-1}}{\gamma+x_{n}}$. Math. Sci. Res. Hot-Line 4, 1-11 (2000)

4. Grove, EA, Kostrov, Y, Ladas, G, Schultz, SW: Riccati difference equations with real period-2 coefficients. Commun Appl. Nonlinear Anal. 14, 33-56 (2007) 
5. Taskara, N, Uslu, K, Tollu, DT: The periodicity and solutions of the rational difference equation with periodic coefficients. Comput. Math. Appl. 62, 1807-1813 (2011)

6. Cinar, C: On the positive solutions of the difference equation $x_{n+1}=x_{n-1} /\left(1+x_{n} x_{n-1}\right)$. Appl. Math. Comput. 150, 21-24 (2004)

7. Papaschinopoulos, G, Papadopoulos, BK: On the fuzzy difference equation $x_{n+1}=A+\frac{B}{x_{n}}$. Soft Comput. 6, 456-461 (2002)

8. Elabbasy, EM, El-Metwally, HA, Elsayed, EM: Global behavior of the solutions of some difference equations. Adv. Differ. Equ. 2011, 28 (2011). doi:10.1186/1687-1847-2011-28

9. Elsayed, EM: Solution and attractivity for a rational recursive sequence. Discrete Dyn. Nat. Soc. 2011, Article ID 982309 (2011)

10. Elsayed, EM: On the solution of some difference equations. Eur. J. Pure Appl. Math. 4, 287-303 (2011)

11. Elsayed, EM: Solutions of rational difference system of order two. Math. Comput. Model. 55, 378-384 (2012)

12. Touafek, N, Elsayed, EM: On the solutions of systems of rational difference equations. Math. Comput. Model. 55 1987-1997 (2012)

13. Elsayed, EM: Behavior and expression of the solutions of some rational difference equations. J. Comput. Anal. Appl. 15(1), 73-81 (2013)

14. El-Metwally, H, Elsayed, EM: Solution and behavior of a third rational difference equation. Util. Math. 88, 27-42 (2012)

15. Koshy, T: Fibonacci and Lucas Numbers with Applications. Wiley, New York (2001)

16. Vajda, S: Fibonacci and Lucas Numbers, and the Golden Section: Theory and Applications. Dover, New York (2007)

17. El Naschie, MS: The golden mean in quantum geometry, Knot theory and related topics. Chaos Solitons Fractals 10(8), 1303-1307 (1999)

18. Marek-Crnjac, L: On the mass spectrum of the elementary particles of the standard model using El Naschie's golden field theory. Chaos Solitons Fractals 15(4), 611-618 (2003)

19. Falcon, S, Plaza, A: The metallic ratios as limits of complex valued transformations. Chaos Solitons Fractals 41, 1-13 (2009)

doi:10.1186/1687-1847-2013-174

Cite this article as: Tollu et al.: On the solutions of two special types of Riccati difference equation via Fibonacci numbers. Advances in Difference Equations 2013 2013:174.

\section{Submit your manuscript to a SpringerOpen ${ }^{\ominus}$ journal and benefit from:}

- Convenient online submission

- Rigorous peer review

- Immediate publication on acceptance

Open access: articles freely available online

- High visibility within the field

- Retaining the copyright to your article 